\section{Arquitectura como percepción}

Patricia Mañana BorRazÁs

Laboratorio de Arqueoloxía, Instituto de Estudios Gallegos Padre Sarmiento (CSIC-XuGa) / UA LaFC (IIT, USC)

\begin{abstract}
Resumen
Este texto trata de ilustrar los tipos de análisis básicos para investigar una cuestión tan intrínsecamente humana como la percepción. En el análisis de una construcción, los aspectos relativos a la percepción nos permiten acceder a una interesante información de cómo una construcción funciona y qué implican sus distintos espacios, información en directa relación con los aspectos más humanos y vivenciales de la arquitectura. Son unos análisis complementarios a los análisis formales, ya que mientras estos nos permiten describir en el espacio y en el tiempo una construcción, los análisis de percepción tratan de incidir en la relación existente entre el ser humano y la construcción, entendiendo a la arquitectura también como elemento físico a experimentar y que ha sido construido para propiciar ciertas percepciones.

Se exponen en primer lugar las claves más básicas de los análisis de percepción y cómo estos se vertebran dentro de una metodología que trata comprender una construcción en todos sus aspectos (formales, sociales y simbólicos), sirviendo como ejemplo su aplicación a un túmulo del neolítico gallego.
\end{abstract}

Palabras Clave: Arqueología de la Arquitectura; Arqueología del Paisaje; Análisis de la percepción; neolítico; túmulo.

\footnotetext{
Abstract

This text sets out to illustrate types of basic analysis in researching a question as intrinsically human as perception. In the analysis of a construction, the aspects relative to perception allow us to access interesting information as to how a construction functions and what is implied by its different spaces, information which is in direct relation to more human, living aspects of architecture. These analyses complement formal analyses, as while these allow us to describe a construction in time and space, analyses of perception seek to stress the existing relationship between the human being and the construction, understanding architecture as a physical element to be experienced and which has been constructed to create a favourable atmosphere for certain perceptions. The study first shows the most basic elements in perception analysis and how these support a methodology that attempts to understand a construction in all its aspects (formal, social, symbolic), giving as an example a Neolithic Galician burial mound.
}

Key words: Archaeology of Architecture; Landscape Archaeology; Perception Analysis; Neolithic; burial mound.

\section{ANALIZANDO LA PERCEPCIÓN}

Se presenta una analítica que se centra en un aspecto muy específico de una construcción: cómo un espacio arquitectónico ha sido construido para propiciar cierta percepción, qué aspectos físicos concretos se advierten y cómo éstos influyen en nuestro movimiento y visión de una determinada arquitectura. Estamos ante unos análisis que son parte de un conjunto de técnicas vertebradas dentro de un plan metodológico, cuyo fin último es acceder a la lógica social y simbólica de dicha construcción y encardinarlo con el resto de evidencias disponibles de esa sociedad.

El análisis de la percepción deber ser un aspecto de la investigación de una arquitectura, no debe ser tomado como único, pues si esto se hace así, se puede llegar a reducir la arquitectura a impresiones, sin tener en cuenta el espacio construido como dimensión existencial-funcional y como relación entre el hombre y el medio que lo rodea. Se parte de la idea de que la organización de los distintos espacios de una construcción, así como la configuración de sus volúmenes se ajusta a un orden perceptivo intencional, el cual es posible reconocer al experimentar los elementos físicos en una secuencia temporal (CHING, 1995). Esto implica estudiar la relación vivencial del ser humano con las construcciones que usa o construye, intentando así acceder al tipo de pensamiento o racionalidad al que responde la forma de organizar tanto las estructuras como los espacios construidos.

\section{LOS ANÁLISIS DE LA PERCEPCIÓN}

La percepción humana se basa en la experimentación de algo en relación con lo que hemos percibido anteriormente. Las técnicas analíticas que aquí se presentan se vertebran en torno a dos acciones relacionadas con la percepción:

-el movimiento, por medio del cual se ha desarrollado el denominado análisis de accesos y de amplia aplicación en los estudios de construcciones desde su aplicación por Faulkner al análisis de castillos y casas escocesas en 1964 (p.e. Hillier, Hanson, 1984; SÁNCHEZ, 1998);

-la percepción visual de los espacios y estructuras, ya que gran parte de la información que recibe el ser humano es de carácter visual y esta información forma parte de la racionalidad del individuo; este aserto ha derivado en los denominados análisis de visibilidad, que han tenido gran desarrollo en la investigación post-procesual (BENDER, 1993; BRADLEY, 1993; Criado, 1988, 1989, 1993; ThOMAS, 1991).

Además de estos dos tipos de análisis, se están desarrollando análisis de percepción ${ }^{1}$ relacionados con las cualidades cromáticas de ciertos elementos, las propiedades

${ }^{1}$ Un volumen recopilatorio de este tipo de aproximaciones es el editado por C. Jones y C. Hayden (1998). 
acústicas, olfativas, ... análisis que nos permiten acceder a una parte importante de la percepción de los espacios pero que no desarrollamos en este trabajo, que se centra únicamente en los aspectos relacionados con cuestiones visuales.

Estos análisis tienen como base el Análisis Formal de la construcción, por medio del cual se han identificado los elementos formales y organizaciones espaciales que se dan en la construcción, y que trata de describir por su propia lógica formal lo que aquí se pretende analizar por su lógica perceptiva, con el fin último de acceder a la racionalidad del grupo que la creó y usó.

\subsection{La percepción en movimiento}

Los análisis de movilidad forman parte de los estudios sintácticos del espacio desarrollados para analizar las relaciones espaciales de una construcción por medio de la circulación entre ellos y el significado social subyacente. Dos son las técnicas fundamentales a emplear en este tipo de análisis: el análisis de circulación y el análisis gamma. El objetivo de estas técnicas es tanto cuantificar la permeabilidad y profundidad de los espacios como identificar el hilo perceptivo de una construcción. Según la metodología ya especificada en un artículo anterior de este volumen (CRIADO, MAÑA$\mathrm{NA}$ ), se deben aplicar en cada ámbito donde se significa la construcción, tanto al nivel del emplazamiento (físico y construido) como en relación a los elementos que componen la construcción.

\subsubsection{Análisis de circulación}

Una de las formas de analizar cómo se percibe un espacio construido es por medio del movimiento hacia él, el recorrido que hacemos tanto para aproximarnos como para pasar de un espacio a otro dentro de la construcción. Es por esta cuestión que preferimos denominar al análisis que tiene como base el movimiento del individuo en un espacio construido análisis de circulación, frente a análisis de accesos, pues no sólo pretendemos valorar el momento de entrada en cada espacio, sino integrarlo en un sistema de tránsito y así poder definir cuáles son los elementos que influyen en la percepción de formas y espacios construidos. Dentro de este tipo de análisis es básica la identificación de ciertos elementos que influyen y dirigen la circulación, como son las escaleras, peldaños, rampas, umbrales, pasillos, caminos, aceras, etc.

Siguiendo al arquitecto F. Ching (1995) en su metodología de análisis arquitectónico, el hilo perceptivo que vincula los distintos espacios en los que se significa la construcción se da en varias fases:

1. la aproximación a la construcción o visión a distancia,
2. ${ }^{\circ}$ el modo de acceso o la entrada al espacio interior,

3. ${ }^{-}$la configuración del recorrido interno,

4. ${ }^{\circ}$ la forma del espacio recorrido

Así, se trata de identificar el hilo perceptivo de una construcción a través del movimiento en sus espacios, reconociendo espacios preeminentes en el esquema general de circulación, bien sea por ser espacios distribuidores o bien porque estos se encuentran al final de un recorrido (SÁNCHEZ, 1998: 102).

\subsubsection{Análsis gamma}

El análisis gamma, que ha sido desarrollado por Hillier y Hanson (1984), se basa en el movimiento a través de los espacios, cuantificando las profundidades y permeabilidades (la facilidad de acceso), valorando el grado de dependencia de unos espacios respecto a otros, el control de acceso y el movimiento que permiten. Los elementos clave en este análisis son los umbrales que separan / comunican los espacios entre sí, pues actúan como controladores de paso a determinado ambiente. Por medio de estos análisis se pueden descubrir las relaciones sociales que mantienen los individuos que habitan una estructura y entre éstos y los foráneos a ella. Su uso para el análisis de los espacios domésticos está muy extendido.

\subsection{El análisis de la percepción visual}

Este análisis trata de identificar el orden perceptivo que se implementa en una construcción, partiendo de la base de que la percepción espacial de una construcción está influida por las cualidades lumínicas, cromáticas, acústicas, de texturas y vistas de los distintos espacios (CHING, 1995).

Las investigaciones que versan sobre la percepción visual de los espacios construidos se fundamentan en la cualidad transespacial de la visión, ya que actúa a distancia, crea una gradación visual según se dispongan los umbrales que, como líneas divisorias entre lo público y lo privado, pueden variar los porcentajes de visibilidad (VEN, 1977). Siendo los umbrales un medio de control de la circulación y también de restricción de la visibilidad, su existencia implica la restricción de ciertas áreas a individuos de una sociedad: no es un espacio público, de libre acceso, sino que sea cual sea el grupo discriminado, hay individuos que se quedan fuera. El análisis de estos aspectos permite «trabajar con aspectos relacionados con la estructura y la ideología de la sociedad» (SÁNCHEZ, 1998: 94).

Básicamente, se llevan a cabo dos tipos de análisis de condiciones de visualización, aunque ambos son complementarios:

- el que se realiza en base a la situación del individuo que percibe, a la visibilidad (es la percepción desde) desde un 
punto de vista determinado, definido normalmente por un umbral y en espacios cerrados (SÁNCHEZ, 1998), o por la ruta de acceso al mismo; trata de identificar el dominio visual de los distintos espacios construidos y el grado de exposición que sufren en relación con el recorrido que se hace a través de ellos, definiendo espacios privados y públicos según el grado de su «exposición a la vista».

- el basado en el impacto visual de los volúmenes y espacios, en la visibilización de los elementos (es la percepción de) y de cómo estos influyen en la percepción; pretende identificar el orden y organización perceptiva de un espacio construido, valorándolo de manera estática en relación tanto a su entorno -el físico / topográfico y el construido (en relación a otras construcciones) - como en relación a la construcción en sí misma. Es fundamental tratar de identificar la voluntad de visibilización (inhibición, ocultación, exhibición y monumentalización, ver CRIADO, 1993: 45-51) de ciertos elementos o espacios dentro de la construcción, hecho que puede ser tanto consciente y explícita como implícita e incluso inconsciente, pero que es fundamental para identificar las cuestiones relacionadas con la parte más simbólica de las construcciones.

Así, con los análisis de percepción visual es posible valorar las estrategias que configuran y ordenan los volúmenes exteriores de una arquitectura: que una zona esté expresamente resaltada puede implicar una cierta estrategia espacial y de percepción que debe ser tenida en cuenta a la hora de describir y valorar una construcción, y hacer explícitos estos aspectos nos pueden permitir determinar qué estrategias de configuración de los espacios se dan en una sociedad, y por lo tanto, acceder a una parte de su patrón de racionalidad.

\section{LA PERCEPCIÓN DEL TÚMULO N.o 5 DEL ALTO DE SAN COSME}

\subsection{Contexto del análisis}

En este apartado vamos a aplicar los análisis anteriormente expuestos a un monumento funerario, la mámoa n. ${ }^{\circ} 3$ de San Cosme (Mos, Pontevedra), yacimiento adscrito al megalitismo gallego, que se encuadra dentro del fenómeno megalítico de la fachada atlántica europea (entre finales del $\mathrm{V}$ milenio y mediados del III milenio a.C.), primera arquitectura monumental del viejo continente y que representa el primer modelo de paisaje histórico basado en construcciones artificiales de carácter permanente. Su excavación ha formado parte del Plan de Control y Corrección del Impacto Arqueológico de la construcción de la Red de Gasificación de Galicia, Red de Vigo-Porriño (AMADo, 2000; PARCERO, 1998).
Como paso previo a las valoraciones que aquí se presentan sobre la percepción del túmulo, se ha llevado a cabo un análisis formal, estratigráfico y espacial (se puede ver de manera detallada en MAÑANA et alli., 2002), en el que se identificó una construcción cuyo volumen y espacio principal es un túmulo en tierra con anillo lítico, estructura que es formalmente disimétrica, ya que mientras la mitad NW es más somera, con menor altura y anillo menos espeso, la SE el túmulo alcanza una mayor altura relativa y el anillo es más amplio y potente. En el espacio interior a éste, y espacialmente dependiente de él, se sitúa una estela de piedra de forma cuadrangular cuya posición responde al mismo eje de organización espacial que el monumento y también es formalmente disimétrico en sus lados menores, ya que mientras la cara NW de la estela es rugosa, la SE es lisa. Un tercer espacio que configura esta construcción es el inmediatamente contiguo al túmulo, en el cual se ha dispuesto una estructura tipo "pavimento" que únicamente se desarrolla al sudeste.

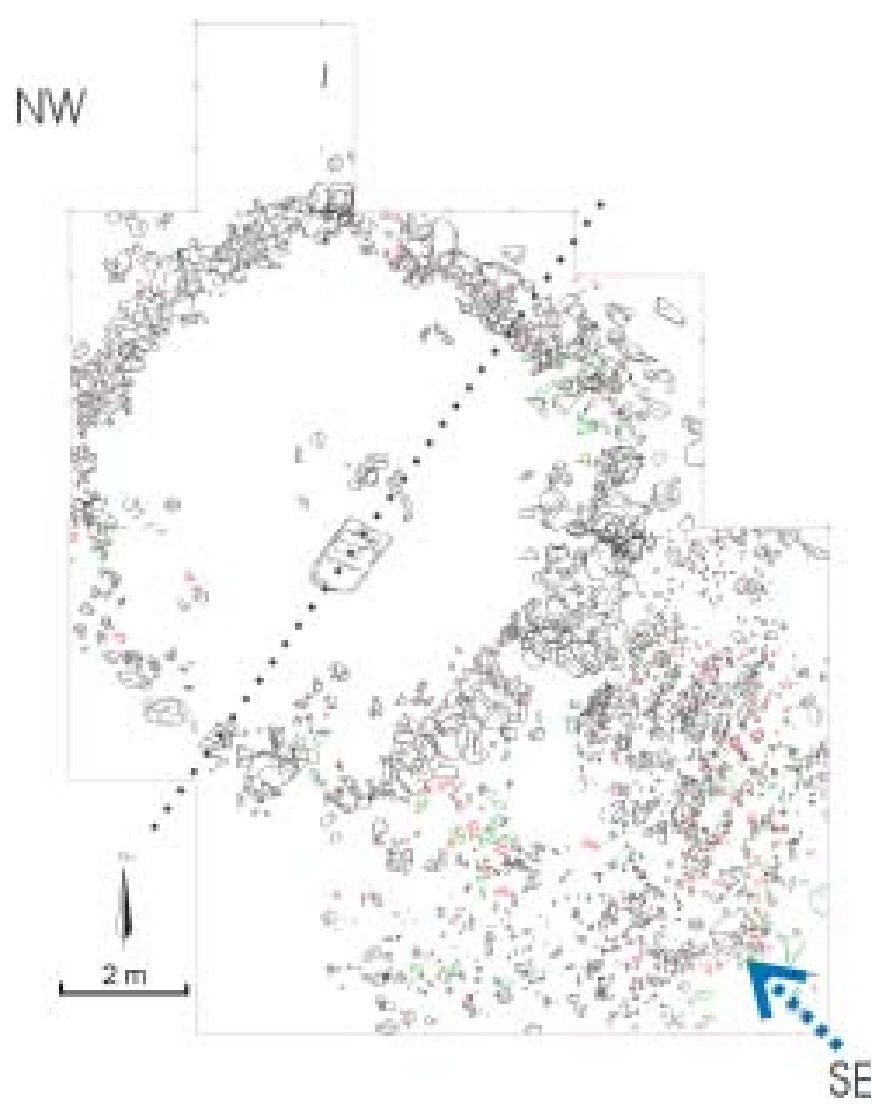

Fig. 1. Planta de las estructuras pétreas del túmulo 3 del Alto de San Cosme 

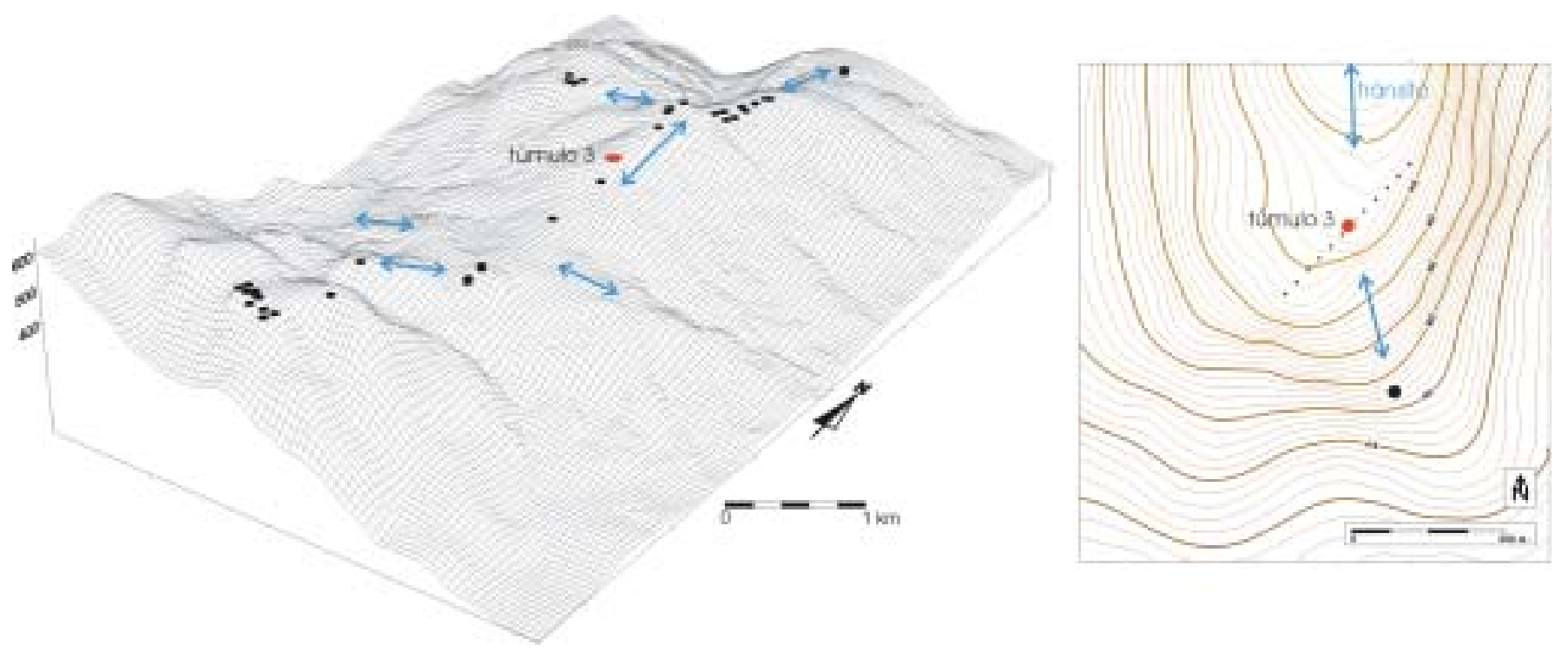

Fig. 2. Mapa de relieve de la zona y del entorno inmediato del túmulo, señalándose la posición de los túmulos y el tránsito general de la zona

\subsection{La percepción del túmulo a través del movimiento}

Para elaborar de forma positiva un análisis de circulación relativo a este yacimiento, primero hay que identificar qué tipo de aproximación al túmulo se puede realizar, para lo que es básica la identificación de las características formales del entorno, tanto las relativas al emplazamiento como en relación con otras construcciones del mismo fenómeno. Por las características formales de la sierra en la que se implanta y en relación con las condiciones más favorables para el movimiento natural, se ha identificado una ruta principal de movimiento lineal de dirección norte-sur.

$\mathrm{Al}$ aproximarnos al monumento entramos en su órbita directa de influencia, el espacio contiguo al túmulo, zona en la que se suelen identificar restos de actividad (deposición de materiales, suelos endurecidos, hogueras, etc.) o el inicio de estructuras de acceso al centro del enterramiento. Entre este espacio exterior y el contiguo no se advierte una ruptura concreta, siendo más implícita que explícita: existe continuidad espacial y visual, pues no hay ningún umbral que los divida, no se ha documentado ningún cambio en el nivel del terreno, etc. El único elemento arquitectónico que existe en este espacio es el pavimento irregular e informe formado básicamente por piedras que son llamativas visualmente (cuarzo y piedras verdes, distintas también al material que más se ha empleado en su construcción), situado en el lado sudeste del conjunto, lo que parece indicar un privilegio de esta área respecto al resto del conjunto.

La única manera de llegar al espacio central del túmulo, en donde se localizan las estructuras intratumulares y la zona de enterramiento, es cruzando por la superficie del túmulo, pues no existe ningún tipo de estructura de acceso intratumular como sí se da en otro tipo de túmulos. El movimiento por la superficie del túmulo no está condicionado por ningún elemento delimitador: es un espacio sin barreras importantes aunque significativamente distinto al entorno inmediato (forma hemisférica, altura superior al entorno y delimitada por el anillo lítico), por lo que el acceso es posible desde todas las áreas del entorno del túmulo. El espacio central (las estructuras intratumulares) tapado por la masa tumular, es un espacio cerrado, de acceso muy restringido, pues únicamente se puede llegar mediante la excavación de un pozo, parece no estar ideado para facilitar su acceso de forma no destructiva para el túmulo.

Como valoración, destacamos el hecho de que la circulación posible en este tipo de construcción es más inducida que obligada, o sea, que salvo para acceder al espacio central una vez concluida la construcción del túmulo, no hay límites físicos al movimiento, no hay ningún pasillo, vano, etc. que limite nuestro movimiento. Lo que sí nos parece reconocer es la intención de influir en el desarrollo del recorrido, de dirigir el movimiento al haberse dedicado un mayor esfuerzo constructivo a la zona sudeste. Así, primero se realizaría la aproximación al monumento en sentido N-S, realizando un recorrido lineal que remata en el centro del túmulo después de atravesar tres espacios significativamente distintos, en el que destaca los cambios de nivel (abajo- arriba-abajo) del recorrido.

Con análisis gamma se puede ver que estamos ante una construcción en la que el paso de un espacio a otro está únicamente controlado por el espacio inmediatamente contiguo. Según la sistematización de Hillier y Hanson (1984: 

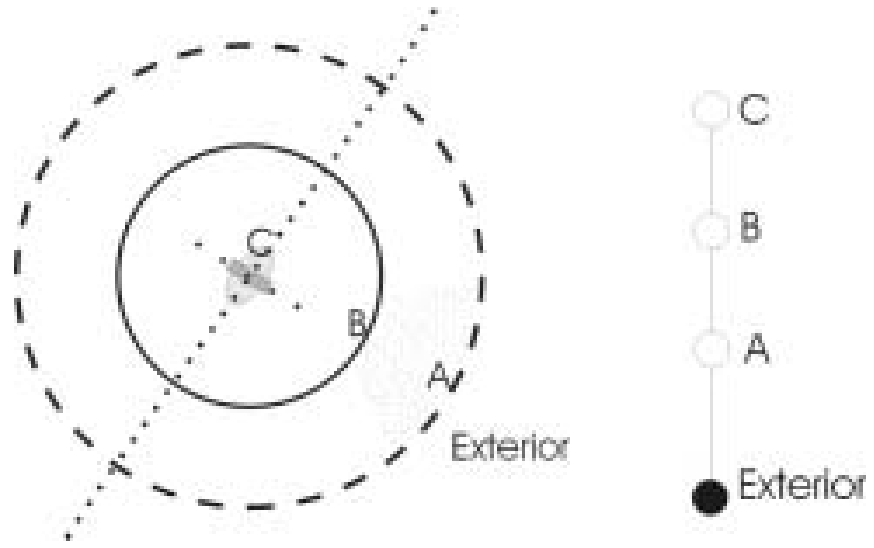

Fig. 3. Gráfico del análisis gamma

94) esto implica que los espacios del túmulo tienen una relación asimétrica, pues para llegar a $C$ (estructuras intratumulares) es necesario pasar antes por $A$ (espacio contiguo) y por $B$ (túmulo).

\subsection{Análisis de las condiciones de visualización}

Los elementos conservados de la configuración original de la arquitectura tumular permiten identificar factores influyentes en la percepción del túmulo: son materiales que por ser diferentes al resto, llamativos tanto por sus propiedades cromáticas (en la composición material de las estructuras, coloraciones significativas en las tierras y piedras) como por sus texturas (contraposiciones entre materiales pétreos y térreos), influyen en el impacto visual del monumento. Así, en este caso nos encontramos ante una construcción que contrapone las texturas y colores de la piedra y la tierra, destacando además el uso restringido de materiales pétreos de color llamativo (piedras verdes y blancas) sobre todo en la mitad sudeste del túmulo.

Se ha identificado que la forma de aproximación preeminente es por el sudeste y es desde este punto desde el que se realiza el análisis de visibilidad. Una de las características de este túmulo en concreto es que apenas destaca sobre su entorno; es un túmulo muy bajo: tendría unos $50 \mathrm{~cm}$. por el lado norte y casi un metro de altura respecto al nivel del suelo en el lado sur, diferencias influidas por la pendiente del emplazamiento. El sector sudeste es la zona en la que el túmulo tiene una mayor altura relativa respecto al nivel del suelo, por lo que adquiere una mayor monumentalidad, logra un mayor impacto visual, coincidiendo además con la zona más destacada formalmente.

Las dimensiones del monumento permiten visualizar desde esta posición casi la totalidad de la superficie del túmulo, recortándose desde esta perspectiva la parte culmi-

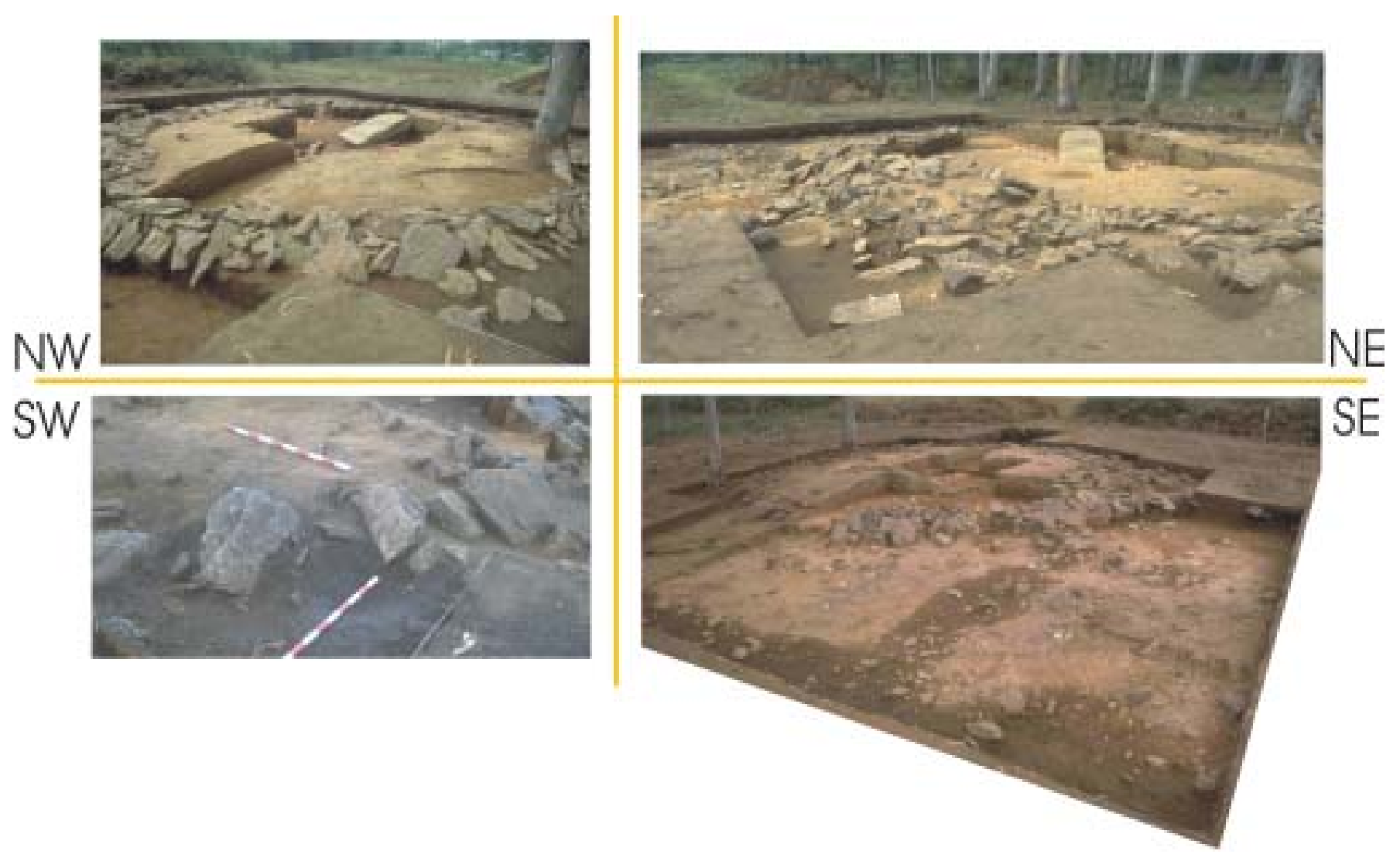

Fig. 4. Vistas del túmulo $n .^{\circ} 3$ del Alto de San Cosme 
nante de todo el anillo lítico que rodea el túmulo, por lo que desde esta posición se alcanza a ver la totalidad del espacio tumular encerrado por el anillo lítico.

En relación a cómo se percibe el entorno del túmulo en relación con este punto de vista, la panorámica se puede describir como constreñida: se realiza contrapendiente y aunque es muy suave, se tiene la percepción cerrada, limitada nuestra visión del entorno inmediato por la zona más alta de la colina, recortándose esta línea de horizonte contra el cielo.

Respecto a las estrategias que se emplean para configurar y ordenar los volúmenes visibles de los elementos arquitectónicos (el análisis de la visibilización), hay que destacar que el resto del túmulo destaca por su sobriedad formal, lo que se ve ampliado por el hecho de que la altura relativa de estos cuadrantes respecto al entorno (sobre todo la del cuadrante noroeste) es nimia. Esta conjunción conlleva que estos sectores casi no tendrían impacto visual si no fuera porque todo el túmulo está rodeado por el anillo lítico, sin percibirse la totalidad del túmulo desde la parte N, y viéndose en su totalidad desde la suroeste, por lo que el túmulo se ve como un espacio restringido, cerrado. Respecto a las perspectivas que se tiene del entorno, desde el sudoeste, la pendiente es algo más suave que por el sudeste, pero aún así la visión del entorno es cerrada aunque sin límites visuales significativos en primer término, abriendo en un segundo término la visión totalmente, recortándose la parte superior de la loma contra el cielo. Desde los sectores septentrionales la perspectiva se reduce al límite del pequeño rellano en el que se sitúa el túmulo, rompiendo la pendiente a escasa distancia al sudeste del túmulo y siendo algo más amplia hacia el suroeste, abriendo en el horizonte la perspectiva visual hacia las sierras del S.

Por lo tanto, se puede comprobar que el túmulo no es visualmente uniforme, sino que tiene diferencias formales que le implementan unos rasgos visuales distintos, lo que se puede relacionar con una estrategia de visualización de los elementos distintiva, pues si su situación y configuración concreta obedece a cierta intención, parece que no se pretende que el conjunto se perciba de forma unitaria. Lo mismo pasa con las perspectivas visuales que desde el túmulo se tienen sobre el entorno, pues mientras en primer término, si la vista se dirige desde la parte sur nos encontraremos con una panorámica cerrada-elevada, si lo hacemos desde el norte, aunque es un ámbito de dominio visual directo es reducido, esta es abierta-descendente y en larga distancia.

Respecto a las relaciones visuales que se manifiestan entre los distintos niveles de articulación espacial del túmulo, en la propia configuración arquitectónica, de los distintos niveles espaciales que se configuran en un túmulo (túmu- lo, espacio intratumular, espacio contiguo), el elemento que adquiere más monumentalidad en el conjunto es el túmulo, tanto por su mayor volumen como por estar (parcialmente) cubierto y limitado por el anillo lítico y por lo tanto también predomina visualmente sobre los otros elementos. La relación visual que mantiene con el espacio intratumular únicamente se articula por medio de la losa central, que probablemente sobresaliera ligeramente del túmulo.

\section{VALORACIÓN FINAL}

El sentido final de estos análisis no puede extraerse únicamente de lo que en ellos se advierte, sino que ha de incardinarse con el restos de evidencias de esta sociedad, como se ha expuesto en otro artículo de esta publicación (CRIADO, MAÑANA): la metodología a la que responden estas técnicas (CRIADO, 1999, 2002; MAÑANA et alli., 2002) se basa en la validación de hipótesis extraídas de las evidencias formales de este fenómeno, la contrastación de lo que en este análisis hemos identificado a nivel de la percepción del espacio construido con lo identificado en otros casos y a otros niveles (emplazamiento, arte, distribución de cultura material, etc).

Los túmulos son la primera construcción de la fachada atlántica europea en la que hay intenciones evidentes de dejar una huella de modificación del entorno: por primera vez se construye con la intención de que lo que se haga, dure. Son espacios construidos monumentales que en contraste con construcciones hechas con materiales más endebles, perecederos, van a permanecer en el paisaje ( $y$ han permanecido hasta nuestros días). Esto contrasta con el modelo de construcción anterior, de las sociedades cazadoras-recolectoras, en las que las evidencias arqueológicas nos hablan de cabañas sencillas o adecuaciones de abrigos, cuevas, hechos con materiales endebles y no mucho trabajo: el uso que estas sociedades hacen de su entorno no deja una huella evidente en él, que se esfuma en un corto periodo de tiempo. En cambio, con las sociedades que construyen túmulos, con una incipiente agricultura, se hacen patentes y evidentes los espacios relacionados con los muertos: los túmulos operan como una tecnología de construcción del paisaje social que domestica el mundo físico a través de dispositivos artificiales, no sólo introduciendo hitos arquitectónicos en el espacio natural para ordenarlo según referencias culturales, sino también controlando e imponiendo un determinado patrón de percepción del entorno a los individuos, una pauta para experimentar el espacio-tiempo comunitario e individual.

Así, por los análisis aquí expuestos parece imponerse un control de la percepción en relación con los túmulos: se crean espacios circulares, eminentemente cerrados y domi- 
nados y centralizados por el propio túmulo como estructura monumental y diferente (opuesto) al entorno natural, un entorno que se advierte disimétrico en sus características formales y perceptivas. Así, el modo de percepción que parece vertebrarse en torno a esta construcción confirma el modelo advertido a escala de organización formal-espacial del fenómeno, cuya valoración ya hemos expuesto en otros puntos de esta misma publicación.

\section{Bibliografía}

Amado Reino X., Mañana Borrazás P., Santos Estévez M., 2000, La Arqueología de la Gasificación en Galicia: Corrección de Impacto de las Redes de Pontevedra, TAPA (Traballos en Arqueoloxía da Paisaxe), n. ${ }^{\circ}$ 18, Santiago de Compostela: Laboratorio de Arqueoloxía e Formas Culturais, ITT, USC.

Ayán Vila X. M., Blanco Rotea R., Mañana Borrazás P. (ed.), 2003, Archaeotecture: Archaeology of Architecture, British Archaeological Reports, International Series, Oxford. En prensa.

Bender J. (ed.), 1993, Landscape, Politics and Perpectives, Oxford.

BRADLEY R., 1993, Altering the Earth. The origins of monuments in Britain and Continental Europe, Society of Antiquaries of Scotland Monograph Series, Number 8, Edimburg.

ChIng F., 1995, Arquitectura: forma, espacio y orden, México.

Criado Boado F., 1988, Arqueología del Paisaje y Espacio Megalítico, Arqueología Espacial, n. ${ }^{\circ}$ 12, pp. 61-117.

Criado Boado F., 1989, Megalitos, Espacio, Pensamiento, Trabajos de Prehistoria n. ${ }^{\circ}$ 46, pp. 75-98.

Criado BoAdo F., 1993, Visibilidad e interpretación del registro arqueológico, Trabajos de Prehistoria, n. ${ }^{\circ}$ 50, pp. 39-56.

Criado Boado F., 1999, Del Terreno al Espacio: Planteamientos y Perspectivas para la Arqueología del Paisaje, CAPA (Criterios y Convenciones en Arqueología del Paisaje), n. ${ }^{\circ}$ 6, Santiago.

Hillier B., Hanson J., 1984, The social logic of space, Cambridge.

Jones C., Hayden C. (ed.), 1998, The Archaeology of Perception and the Senses, Archaeological Review from Cambridge, n. ${ }^{\circ} 15$ (1).

mañana Borrazás P., Blanco Rotea R., Ayán Vila J.M., 2002, Arqueotectura 1: Bases teórico-Metodológicas para una Arqueología de la Arquitectura, TAPA (Traballos en Arqueoloxía da Paisaxe), n. ${ }^{\circ}$ 15, Santiago de Compostela.

PARCERO OUBINÁA C., 1998, La arqueología en la gasificación de Galicia: Excavación del Túmulo n. 3 del Alto de San Cosme, TAPA (Trabajos en Arqueología del Paisaje), n. ${ }^{\circ}$ 5, Santiago de Compostela.

SÁnCHEZ J., 1998, La Arqueología de la Arquitectura. Aplicación de nuevos modelos de análisis a estructuras de la Alta Andalucía en época ibérica, Trabajos de Prehistoria, n. ${ }^{\circ}$ 55(2), pp. 89-109.

THOMAS J., 1991, Rethinking the Neolithic, Cambridge.

VEN VAN DE C., 1981, El espacio en arquitectura. La evolución de una idea nueva en la teoría e historia de los movimientos modernos, Madrid. 